face, frontal bossing, and low-set protruding ears. His neurologic examination revealed normal muscular tonus and normal deep tendon reflexes with clumsy gait. Metabolic screening tests, including tandem mass, urine organic acids, plasma, and urine amino acid profiles were also normal. The patient underwent MRI of the brain as a firstline investigation, and T2 images demonstrated an extensive involvement of the hemispheric, subcortical white matter with a cerebrospinal fluid intensity signal change suggestive of multilocular giant VRS. MR spectroscopy showed no significant signal peak. The karyotype analysis of the patient was $46 \mathrm{XY}$, and FISH for Angelman syndrome also revealed a normal result. In the 2-year follow-up, the patient showed no neuromotor deterioration and radiological progression.

In conclusion, VRS must be differentiated from other cystic lesions of the brain. Phenotypic characteristics of our patient were not compatible with the previously defined syndromes.

\section{PO-0807 LONG TERM NEURODEVELOPMENTAL OUTCOME OF PRETERM INFANTS WITH PERIVENTRICULAR-INTRAVENTRICULAR HAEMORRHAGE}

E Bayram, M Torun Bayram, Y Topcu, S Hiz. Pediatrics, Dokuz Eylul University Hospital, Izmir, Turkey

\subsection{6/archdischild-2014-307384.1441}

Background and aims To determine the neurodevelopmental morbidity of preterm infants with periventricular intraventricular haemorrhage, at the age of 4 .

Methods The patients at the age of 4 were evaluated through neurologic examination and motor assessment by a paediatric neurologist and Denver II Developmental Screening Test by a psychologist. The results were compared with Denver II Developmental Screening Test results which had been made at 3-6 and 6-12 months.

Results The total study population consisted of 66 prematurely born children of less than 37 gestational age. When the cases with PVH-IVH were graded with the cranial neuroimaging findings, $62,1 \%$ were documented as grade I haemorrhage, $18 \%$ as grade II, $6 \%$ as grade III and $13.9 \%$ as grade IV. Patients with grade III-IV Periventricular Intraventricular Haemorrhage had significantly lower Denver II Developmental Screening Test results at the age of 4, compared with grade I-II PeriventricularIntraventricular Haemorrhage group. Similarly, $\leq 32$ weeks patients had significantly lower Denver II Developmental Screening Test at the age of 4 when compared with $>32$ weeks patients.

Conclusions Children who were born $\leq 32$ gestational weeks and/or patients with grade III-IV periventricular-intraventricular Haemorrhage have an increased risk of neurologic impairment. All premature infants should be evaluated by Denver II Developmental Screening Test in early childhood period of life.

\section{PO-0808 THE AFFECT OF APOE GENE POLYMORPHISM ON NEURO-COGNITIVE FUNCTIONS IN CHILDREN WITH CONGENITAL HEART DISEASE}

${ }^{1} \mathrm{~T}$ Bedir Demirdag, ${ }^{1} \mathrm{~K}$ Gucuyener, ${ }^{1}$ AS Soysal, ${ }^{2} \mathrm{NS}$ Guntekin, ${ }^{1} \mathrm{Z}$ Ozturk. ${ }^{1}$ Pediatrics, Gazi University Faculty of Medicine, Ankara, Turkey; ${ }^{2}$ Genetics, Gazi University Faculty of Medicine, Ankara, Turkey

10.1136/archdischild-2014-307384.1442
Research conducted on children with CHD displays that these children's neurological development is different than the normal population and focuses on the reasons of this difference. Currently, the factor that attains the highest emphasis is the Apo E genotype of the patients. We aimed at revealing the influence of Apo E gene on the neurological development process of children with CHD. Our goal is, predicting the nurological development of children with CHD according to Apo E gene expression, and anchoring the children requiring support, at an earlier stage. We investigated 188 children patients with CHD, in GUTF paediatric cardiology departmentt, between 2009-2013. We documented the socio-demographic parameters. After physical examinations followed by psychometric tests, we examined Apo E genotype on blood samples of the children. Cyanotik patients' motor functions were worse then acyanotic patients $(p<0,05)$. Patients with VSD got higher points from the WISCR total IQ, compared to the patients who do not have SD. The other psychometric tests on the children did not display any further difference. $78.7 \%$ of the patients who were involved in our research classify as E3/E3. Sociocultural and economic status of parents was positively associated with psychometric test results $(\mathrm{p}<$ 0,05).

Relevant literature claim that children with CHD display worse neurocognitive functioning compared to normal population, and having Apo E2 allel is a risk factor for it. Apo E4 allel is more related to better psychometric test.

Results However, our results display no influence of ApoE gene on the neurocognitive functioning.

\section{PO-0809 SOCIAL COMMUNICATION QUESTIONNAIRE FACTOR STRUCTURE FOR PRIMARY SCHOOL CHILDREN WITH DEVELOPMENTAL DISORDERS SCREENED AS PART OF AN AUTISM PREVALENCE STUDY}

AM Boilson, A Staines, MR Sweeney. Nursing \& Human Sciences, Dublin City University, Co. Dublin, Ireland

\subsection{6/archdischild-2014-307384.1443}

Background and aims Explore the factor structure of the Social Communication Questionnaire (SCQ: Rutter et al., 2003) for primary school children 6-11 years, 7951 screened as part of an autism prevalence study identified with diagnosed developmental disorders including Autism Spectrum Disorders (ASDs).

Methods The SCQ is a 40-item parent report questionnaire that asks about characteristic autistic behaviour. It is based on the Autism Diagnostic Interview - Revised (ADI-R: Lord et al, 1994) recommended cut off score for ASDs $(>15)$. Sixty nine percent 5457 of parents completed the SCQ, 7\% 411 were identified (males 294, 71\%) with parent reported diagnosed developmental disorders: Speech and language 227, 55\%; ADHD 64, 16\%; ASDs 58, 14\%; Dyspraxia 49, 12\%; Downs Syndrome 8, $2 \%$ other diagnosis $5,1 \%$.

Results The optimal SCQ cut off score for differentiating ASDs from other developmental disorders was $(>13)$ sensitivity 0.89 , specificity 0.81, PPV 0.43, NPV 0.98. Principal Components Analysis revealed 4 factors explaining $49 \%$ of the total variance. First factor, 32\%: 7 of 10 items, Reciprocal Social Interaction (RSI) ADI-R domain. Second factor 9\%, 6 of 8 Restricted Repetitive Stereotyped Behaviour (RRSB) domains third and fourth factors each explaining 4\% variation. Four of 6 item's on the third factor (RSI) domain, 4 of 5 on the fourth ADI-R Communication domain. 
Conclusions A substantial number of communication items did not correlate well with the total score. These items should be revised for screening in a general population setting. Discriminative ability of the SCQ total score performed well for differentiating children with ASDs from other developmental disorders.

\section{P0-0810 PARENTAL SOCIO-ECONOMIC CHARACTERISTICS AND SOCIAL COMMUNICATION QUESTIONNAIRE SCORES FOR PRIMARY SCHOOL CHILDREN SCREENED AS PART OF AN AUTISM PREVALENCE STUDY}

AM Boilson, A Staines, MR Sweeney. Nursing \& Human Sciences, Dublin City University, Co. Dublin, Ireland

\subsection{6/archdischild-2014-307384.1444}

Background and aims To describe SCQ scores based on parental socio-economic characteristics for primary school children 6-11 years, 7951 screened as part of an autism prevalence study in three urban regions of the Republic of Ireland (Galway, Waterford, Cork).

Methods A study booklet was completed by parents of eligible children included: demographics, developmental history, and a screening instrument, Social Communication Questionnaire Lifetime Form (SCQ: Rutter et al., 2003).

Results The majority of study booklets were completed by mothers 4,474 (86\%). Overall the highest mean total SCQ scores were for mothers of children educated to primary or secondary level education $42 \%, 2195 ; 5.54 \pm 4.98$ working in skilled and semi skilled manual occupations 25\%, 807; $5.44 \pm$ 4.63 or described their ethic cultural background as Irish traveller other white background $52811 \%$; $5.97 \pm 5.34$, African other black background $301,6 \% ; 6.72 \pm 50.2$. There were no significant differences in mean SCQ scores where mothers expressed concerns at any stage of their child's development with and without diagnosed developmental disorders in relation to mothers educated to primary or secondary level education, working in skilled and semi skilled manual occupations, or those who described their ethic cultural background as Irish traveller other white background.

Conclusions Mother's first language and level of education may have been contributing factors relating to a proportion of observed high scores. However a number of the mothers of these children who expressed developmental concerns at any stage of the child's development required further screening and/ or referral for ADOS/ADI-R assessment.

\section{PO-0811 CAT-SCRATCH DISEASE: STILL A CHALLENGE}

${ }^{1} \mathrm{~S}$ Bota, ${ }^{2} \mathrm{~S}$ Jacinto, ${ }^{3} \mathrm{C}$ Ferreira, ${ }^{4} \mathrm{AS}$ Santos, ${ }^{1} \mathrm{~L}$ Varandas, ${ }^{1} \mathrm{C}$ Gouveia. ${ }^{1}$ Pediatric Infecciology Unit, Hospital Dona Estefânia CHLC, Lisboa, Portugal; ' ${ }^{2}$ Pediatric Neurology Unit, Hospital Dona Estefânia CHLC, Lisboa, Portugal; ${ }^{3}$ Ophtalmology Unit, Hospital Dona Estefânia CHLC, Lisboa, Portugal; ${ }^{4}$ Center for Vectors and Infectious Diseases Research, National Institute of Health Dr Ricardo Jorge, Águas de Moura, Portugal

10.1136/archdischild-2014-307384.1445

Introduction Cat-scratch disease (CSD)-neuroretinitis (CSDN) is an unusual pathology in immunocompetent individuals (1-2\% of CSD). It typically presents with sudden vision loss following a febrile illness.
Case report A 10 year-old healthy girl presented with fever, headache, vomits and abdominal pain for $48 \mathrm{~h}$. Also, she referred myodesopsias on her right eye. She had daily contact with kittens. Physical examination showed bilateral cervical nodes. At presentation, her ophthalmologic examination revealed a right eye disc swelling with normal visual acuity. One-week later, unilateral sudden diminished visual acuity $(\mathrm{RE}=2 / 10)$ with macular serous detachment was detected.

Blood analysis revealed normal white cell count, increased Creactive protein $(138 \mathrm{mg} / \mathrm{L})$ and erythrocyte sedimentation rate (44 mm/h). A lumbar puncture showed pleocytosis (56/uL). Cranial and orbit MRI were normal. Blood and LCR cultures were negative. HIV, herpesviruses, Toxoplasma gondii, Mycoplasma pneumoniae, Borrelia burgdorferi, Mycobacterium tuberculosis, syphilis and autoimmune diseases were excluded.

Serologic test (IFA) for Bartonella spp, in blood and CSF, was positive (antibodies titers: IgM 32; IgG 4096) with negative DNA amplification. She was treated with doxycycline and rifampicin. Moreover, intravenous corticosteroids were added in the first 3 days. She became asymptomatic and right visual acuity was $10 / 10$ after 3 months.

Discussion The absence of a macular star does not exclude and should not delay the diagnosis of CSDN. Serology remains the most common test to support the diagnosis. The role of antibiotics with or without steroids is not consensual. Still, patients usually have a favourable visual outcome.

\section{PO-0812 NEURODEVELOPMENTAL OUTCOMES OF PREMATURE INFANTS WITH SEVERE INTRAVENTRICULER HEMORRHAGEA}

E Calisici, Z Eras, IK Gokce, MY Oncel, SS Oguz, U Dilmen. Neonatology, Zekai Tahir Burak Maternity Teaching Hospital, Ankara, Turkey

\subsection{6/archdischild-2014-307384.1446}

Objective Our objective was to determine the neurodevelopmental outcome at 18-24 months' of corrected age (CA) in preterm infants with severe intraventricular haemorrhage (IVH).

Methods This was a retrospective cohort study of all preterm infants who were <37 weeks' gestation, had Grade 3-4 IVH. A comprehensive assessment including hearing, vision, neurological and developmental evaluation with Bayley Scales of Infant Development, Second edition (BSID II) was performed by the experienced researchers at 18 to 24 months' CA.

Results A total of 138 were diagnosed as severe IVH (Grade 34). Median Apgar score $(\mathrm{p}<0.01)$ and resuscitation at birth $(\mathrm{p}<$ 0.01 ) were significantly different for group 1 , group 2 and group 3. The use of catheterization, need for mechanical ventilation, need for phototherapy, retinopathy of premature and bronchopulmonary dysplasia were significantly higher in-group 1 compared to group 2 and 3 ( $\mathrm{p}<0.001, \mathrm{p}<0.001, \mathrm{p}<0.01, \mathrm{p}$ $<0.01$ and $\mathrm{p}=0.014$ respectively). The duration of hospitalisation and mortality rates consistent with the degree of prematurity and were significantly higher in-group 1 compared to group 2 and $3(\mathrm{p}=0.03$ and $\mathrm{p}=0.01)$. Among the long-term outcomes; the rates of CP and NDI did not differ between the groups $(\mathrm{p}=0.68$ and $\mathrm{p}=0.068)$.

Conclusion Our results demonstrated that long-term outcomes of preterm infants did not differ between the groups classified according to the birth weight at 2 years of age. This has leaded to the conclusion that severe IVH is alone represents a 